Article

\title{
Consumption of Low-Calorie Sweeteners among U.S. Adults Is Associated with Higher Healthy Eating Index (HEI 2005) Scores and More Physical Activity
}

\section{Adam Drewnowski * and Colin D. Rehm}

Center for Public Health Nutrition, University of Washington, Box 353410; Seattle, WA 98195, USA;

E-Mail: crehm@uw.edu

* Author to whom correspondence should be addressed; E-Mail: adrewnow@fhcrc.org; Tel.: +206-543-8016; Fax: +206-685-1696.

Received: 11 August 2014 / Accepted: 26 September 2014 / Published: 17 October 2014

\begin{abstract}
The possibility that low-calorie sweeteners (LCS) promote lower quality diets and, therefore, weight gain has been noted as a cause for concern. Data from a representative sample of 22,231 adults were obtained from five cycles of the National Health and Nutrition Examination Survey (1999-2008 NHANES). A single 24-hour recall was used to identify consumers of LCS beverages, foods and tabletop sweeteners. Diet quality was assessed using the Healthy Eating Index 2005 (HEI 2005) and its multiple subscores. Health behaviors of interest were physical activity, smoking and alcohol use. LCS consumers had higher HEI 2005 scores than did non-consumers, largely explained by better SoFAAS subscores (solid fats, added sugar and alcohol). LCS consumers had better HEI subscores for vegetables, whole grains and low-fat dairy, but worse subscores for saturated fat and sodium compared to non-consumers. Similar trends were observed for LCS beverages, tabletop LCS and LCS foods. Consumers of LCS were less likely to smoke and were more likely to engage in recreational physical activity. LCS use was associated with higher HEI 2005 scores, lower consumption of empty calories, less smoking and more physical activity.
\end{abstract}

Keywords: low-calorie sweeteners; diet quality; Healthy Eating Index 2005; health behaviors 


\section{Introduction}

Replacing added sugars in beverages and foods with low-calorie sweeteners (LCS) is one way to manage body weight [1-4]. Overweight adults are more likely to consume LCS products than are normal-weight adults [5]. Combining LCS use with higher-quality diets and with more physical activity would be an even more comprehensive approach to weight control [6,7].

Based on existing data, the consumers of diet products in the U.S. are more likely to be non-Hispanic white women with higher education and incomes [8]. The consumption of diet beverages in the U.S. is higher among groups of higher socioeconomic status (SES), as is the consumption of bottled and tap water [9]. Diet quality, as measured by Healthy Eating Index (HEI 2005) scores, also tends to be higher among individuals of higher SES [10]. Physical activity and smoking follow similar socio-demographic trends [11,12].

Concerns about the use of LCS for weight control [4] have invoked the possibility that the use of sweet, yet non-caloric LCS products might confuse the body, provoke increased appetite for sweet foods, reduce overall diet quality and contribute to weight gain. The present hypothesis was that, to the contrary, LCS use would be associated with better-quality diets and with more positive health behaviors, after adjusting for covariates [6,13].

LCS consumers were stratified by product type: LCS beverages, tabletop LCS and LCS foods [14]. Although diet beverages are the category leader, tabletop LCS and LCS foods also play a role in weight control [15]. Diet quality was assessed using the Healthy Eating Index (HEI 2005), a tool developed by the U.S. Department of Agriculture to measure compliance with U.S. dietary recommendations and guidelines [16]. Among HEI subscores, the consumption of solid fats, alcohol and added sugars (SoFAAS subscore) was of particular interest. Any increase in added sugars consumption would be reflected in a less favorable SoFAAS subscore [5].

\section{Experimental Section}

\subsection{Population Sample}

The National Health and Nutrition Examination Survey (NHANES) provides data on dietary intakes and multiple health indicators for a nationally-representative sample of children and adults in the United States [17]. The present analyses used data from 5 NHANES cycles: 1999-2000, 2001-2002, 2003-2004, 2005-2006 and 2007-2008. Included in the analysis were data for 22,231 adults ( $\geq 20$ years), who were not pregnant, for whom height and weight data were available and who completed a 24-h dietary recall. All study protocols for NHANES 1999-2008 were approved by the institutional review board at the National Center for Health Statistics [18], and informed consent was provided by all participants.

Dietary collection methods differed depending on the cycle of NHANES. NHANES 1999-2002 collected data using a computer-assisted dietary interview system (CADI), whereas the later cycles (NHANES 2003-2008) used the USDA Automated Multiple Pass Method, administered by trained interviewers, to obtain 24-h recalls. Respondents reported the types and amounts of all food and beverages consumed in the preceding 24-h, from midnight to midnight. The detailed methodology has been reported elsewhere [17,19]. Further, NHANES 1999-2002 collected only one 24-h recall, whereas 
the later cycles collected 2 recalls, one in person and one by telephone. The present analyses were based on the first 24-h recall. A single 24-h recall for a large population yields an unbiased estimate of the dietary patterns of populations. Participant characteristics, including age, gender, education and race/ethnicity, were self-reported.

\subsection{Classification of LCS Consumption by Product Category}

The Food and Nutrition Database for Dietary Studies [20] used to calculate energy and nutrient intakes in NHANES does not formally code foods and beverages containing LCS. To develop a custom coding algorithm, we examined approximately 5700 items in the individual foods' file to identify those foods and beverages that did contain LCS. Individual foods and beverages were queried based on the food description, energy density (kcal/100 g) and total and added sugars content (g) per average consumption report.

The most common LCS beverages were "soft drink, cola-type, sugar-free”, "soft drink, cola-type, decaffeinated, sugar-free”, "soft drink, fruit-flavored, sugar-free, caffeine free” and "fruit-flavored drink, made from powder, low calorie”. The LCS designation also included teas pre-sweetened with LCS. The most frequently used tabletop LCS were saccharin, sucralose and aspartame. Liquid LCS were also included in this category, but represented a very small number of consumption reports compared to powder-based LCS. Key LCS foods included yogurt, ice cream, grain-based desserts and candies. The level of detail in the food database did not permit for the evaluation of specific types of LCS (e.g., comparison of sucralose vs. saccharin).

NHANES participants were then assigned to 4 different categories of LCS consumers, namely: (1) consumers of LCS beverages (e.g., diet soft drinks, diet fruit drinks, diet iced tea and low-calorie energy drinks); (2) consumers of LCS foods (e.g., yogurt, ice cream, baked goods or candies); (3) consumers of tabletop LCS (e.g., sucralose, aspartame or saccharin); and (4) consumers of any LCS from any source (beverages, foods or tabletop).

\subsection{Diet Quality Measures and Health Behaviors}

Diet quality was assessed using the Healthy Eating Index (HEI 2005), a tool developed by the U.S. Department of Agriculture to measure compliance with dietary recommendations and guidelines. The HEI 2005 is a 12-component 100-point scale that assesses the adequacy and moderation components of the American diet. Higher scores are associated with better dietary compliance $[10,16]$. Physical activity was assessed through a self-reported questionnaire asking about various moderate and vigorous physical activities. Metabolic Equivalent of Tasks (MET) values were estimated from the physical activity questionnaire. Smoking status was categorized into: current, former or never, based on self-report. Alcohol use was assessed through both the 24-h recall and an alcohol use questionnaire, which was used to assess habitual alcohol use (e.g., average number of drinks per week). 


\subsection{Statistical Analysis}

Descriptive analyses compared LCS consumers and non-consumers separately for each product category (beverage, tabletop, food). For all analyses, except those by age group, estimated proportions were adjusted for age group using direct standardization. Statistical heterogeneity was evaluated with a survey-weighted Wald test. In analyses adjusted for age group, gender and race/ethnicity a survey-weighted linear (for continuous outcomes like HEI 2005) or multinomial logistic (for categorical outcomes like physical activity) regression model was fit. The survey-weighted marginal means/proportions were then estimated, which can be considered estimates of the age, gender and race-adjusted means/proportions. Data analysis was conducted using Stata 11 (College Station, TX, United States). All analyses accounted for the complex survey-design of NHANES data and are representative of the U.S. population.

\section{Results}

The socio-demographic variables for any LCS consumers and non-consumers are shown in Table 1. LCS consumers were more likely to be older, female, non-Hispanic whites, born in the United States and with higher education and incomes. The effects of all of the socio-demographic variables were significant. The age range for peak LCS consumption was 45-74 years.

The socioeconomic gradient was strong: LCS were consumed by $17.3 \%$ of persons with a $<9$ th grade education and by $37.4 \%$ of persons with a college education. LCS consumption almost doubled from the lowest to highest categories of the family income-to-poverty ratio.

Table 1. Age-adjusted proportions consuming any low-calorie sweetener (LCS) (and standard errors) by socio-demographic group, National Health and Nutrition Examination Survey (NHANES) 1999-2008.

\begin{tabular}{ccccc}
\hline & $\boldsymbol{n}$ & Any LCS & No LCS & P-difference \\
\hline Total & 22,231 & $30.0(0.6)$ & $70.0(0.6)$ & - \\
\hline Age group & & & & \\
$20-24$ & 1860 & $12.5(1.1)$ & $87.5(1.1)$ & \\
$25-34$ & 3430 & $21.0(1.0)$ & $79.0(1.0)$ & \\
$35-44$ & 3853 & $29.8(1.2)$ & $70.2(1.2)$ & \\
$45-54$ & 4001 & $35.7(1.1)$ & $64.3(1.1)$ & $<0.001$ \\
$55-64$ & 3057 & $39.4(1.2)$ & $60.6(1.2)$ & \\
$65-74$ & 3140 & $39.3(1.2)$ & $60.7(1.2)$ & \\
$75+$ & 2890 & $30.6(1.2)$ & $69.4(1.2)$ & \\
Gender & & & & \\
Female & 11,046 & $34.0(0.8)$ & $66.0(0.8)$ & $<0.001$ \\
Male & 11,185 & $25.5(0.7)$ & $74.5(0.7)$ & \\
\hline
\end{tabular}


Table 1. Cont.

\begin{tabular}{ccccc}
\hline Race/ethnicity & & & & \\
Mexican-American & 4543 & $23.4(0.8)$ & $76.6(0.8)$ & \\
Other Hispanic & 1288 & $21.8(1.7)$ & $78.2(1.7)$ & \\
Non-Hispanic white & 11,071 & $33.2(0.8)$ & $66.8(0.8)$ & $<0.001$ \\
Non-Hispanic black & 4541 & $18.8(0.8)$ & $81.2(0.8)$ & \\
Other race/multi-racial & 788 & $22.5(1.8)$ & $77.5(1.8)$ & \\
\hline Family income-to-poverty ratio & & & & \\
0-0.99 & 3756 & $19.6(0.9)$ & $80.4(0.9)$ & \\
1.0-1.99 & 5432 & $23.2(1.0)$ & $76.8(1.0)$ & \\
2.0-2.99 & 3347 & $29.2(1.4)$ & $70.8(1.4)$ & \\
3.0-3.99 & 2454 & $33.3(1.4)$ & $66.7(1.4)$ & \\
$\geq 4.0$ & 5489 & $35.8(1.0)$ & $64.2(1.0)$ & \\
Education (age $\geq 25)$ & & & & \\
$<9$ th grade & 3092 & $17.3(1.1)$ & $82.7(1.1)$ & \\
9-11th grade & 3257 & $23.2(1.1)$ & $76.8(1.1)$ & \\
High-school graduate & 4788 & $29.7(1.0)$ & $70.3(1.0)$ & \\
Some college & 5019 & $34.0(1.0)$ & $66.0(1.0)$ & \\
College graduate & 3854 & $37.4(1.3)$ & $62.6(1.3)$ & \\
\hline Place of birth & & & & \\
United States & 17,253 & $31.5(0.6)$ & $68.5(0.6)$ & \\
Mexico & 2669 & $17.1(1.1)$ & $82.9(1.1)$ & \\
Elsewhere & 2302 & $19.7(1.4)$ & $80.3(1.4)$ & \\
\hline
\end{tabular}

${ }^{1}$ Not age adjusted; LCS: low-calorie sweeteners.

\subsection{LCS Use and the Healthy Eating Index (HEI 2005)}

Table 2 shows that LCS consumers had significantly higher HEI 2005 scores than did non-consumers, while Figure 1 and Table A1 shows the HEI 2005 subscores for LCS consumers and non-consumers. The association held for consumers of any LCS and for users of LCS beverages, tabletop LCS and for LCS foods ( $p<0.001$ ) (see Figure 2 and Table A2). The association was largely driven by lower calories from SoFAAS; differences in SoFAAS scores were significant for LCS beverages, for tabletop LCS and for LCS foods $(p<0.001)$. Once energy from SoFAAS were removed from the analysis, the differences in HEI scores were not significant for LCS beverages. However, differences in the total score (minus SoFAAS) were still significant for users of tabletop LCS and LCS foods.

Figure 2 and Table A2 show that users of LCS foods had the highest total HEI-2005 scores. Compared to non-users of LCS foods, this group had significantly higher subscores on total fruit $(p<0.004)$, whole fruit ( $p<0.001)$, dark green/orange vegetables and legumes $(p<0.001)$, low fat dairy $(p<0.001)$ and meat and beans $(p<0.005)$. Users of tabletop LCS had significantly higher subscores on total vegetables $(p<0.001)$, dark green/orange vegetables and legumes $(p<0.001)$, whole grains $(p<0.001)$ and meat and beans $(p<0.005)$ compared to non-consumers. Consumers of LCS beverages had higher HEI 2005 subscores for total vegetables ( $p<0.001)$, whole grains $(p<0.001)$, milk/dairy $(p<0.001)$ and meat and beans $(p<0.001)$ compared to non-consumers. Consumers of any LCS also tended to have better oils subscore (i.e., higher intake) $(p<0.001)$, though the difference was not significant for LCS foods. 
Table 2. Adjusted associations between low-calorie sweetener (LCS) use by product category and Healthy Eating Index (HEI 2005) score and SoFAAS ${ }^{1}$ subscore. Analysis adjusted for age group, gender and race/ethnicity. NHANES 1999-2008.

\begin{tabular}{cccc}
\hline & HEI 2005 & SoFAAS $^{\mathbf{1}}$ & HEI (no SoFAAS) ${ }^{\mathbf{1}}$ \\
\hline Any LCS & $53.6(0.3)$ & $11.1(0.1)$ & $42.5(0.2)$ \\
No LCS & $50.4(0.3)$ & $8.3(0.1)$ & $42.1(0.2)$ \\
$P$-difference & $<0.001$ & $<0.001$ & 0.10 \\
LCS beverages & $54.3(0.4)$ & $11.9(0.2)$ & $42.3(0.3)$ \\
No LCS beverages & $50.7(0.3)$ & $8.5(0.1)$ & $42.2(0.2)$ \\
$P$-difference & $<0.001$ & $<0.001$ & 0.69 \\
\hline Tabletop LCS & $53.8(0.4)$ & $10.9(0.2)$ & $42.9(0.3)$ \\
No tabletop LCS & $51.1(0.3)$ & $8.9(0.1)$ & $42.1(0.2)$ \\
$P$-difference & $<0.001$ & $<0.001$ & 0.003 \\
LCS foods & $57.1(0.7)$ & $12.3(0.3)$ & $44.8(0.5)$ \\
No LCS foods & $51.1(0.2)$ & $9.0(0.1)$ & $42.1(0.2)$ \\
$P$-difference & $<0.001$ & $<0.001$ & $<0.001$ \\
\hline
\end{tabular}

${ }^{1}$ Calories from solid fats, alcohol and added sugars (SoFAAS); LCS: low-calorie sweeteners.

Figure 1. Adjusted association between any low-calorie sweetener (LCS) consumption and mean Healthy Eating Index 2005 (HEI) subscores (maximum value indicated in parentheses). Analysis adjusted for age group, race/ethnicity and gender. Error bars are standard errors.

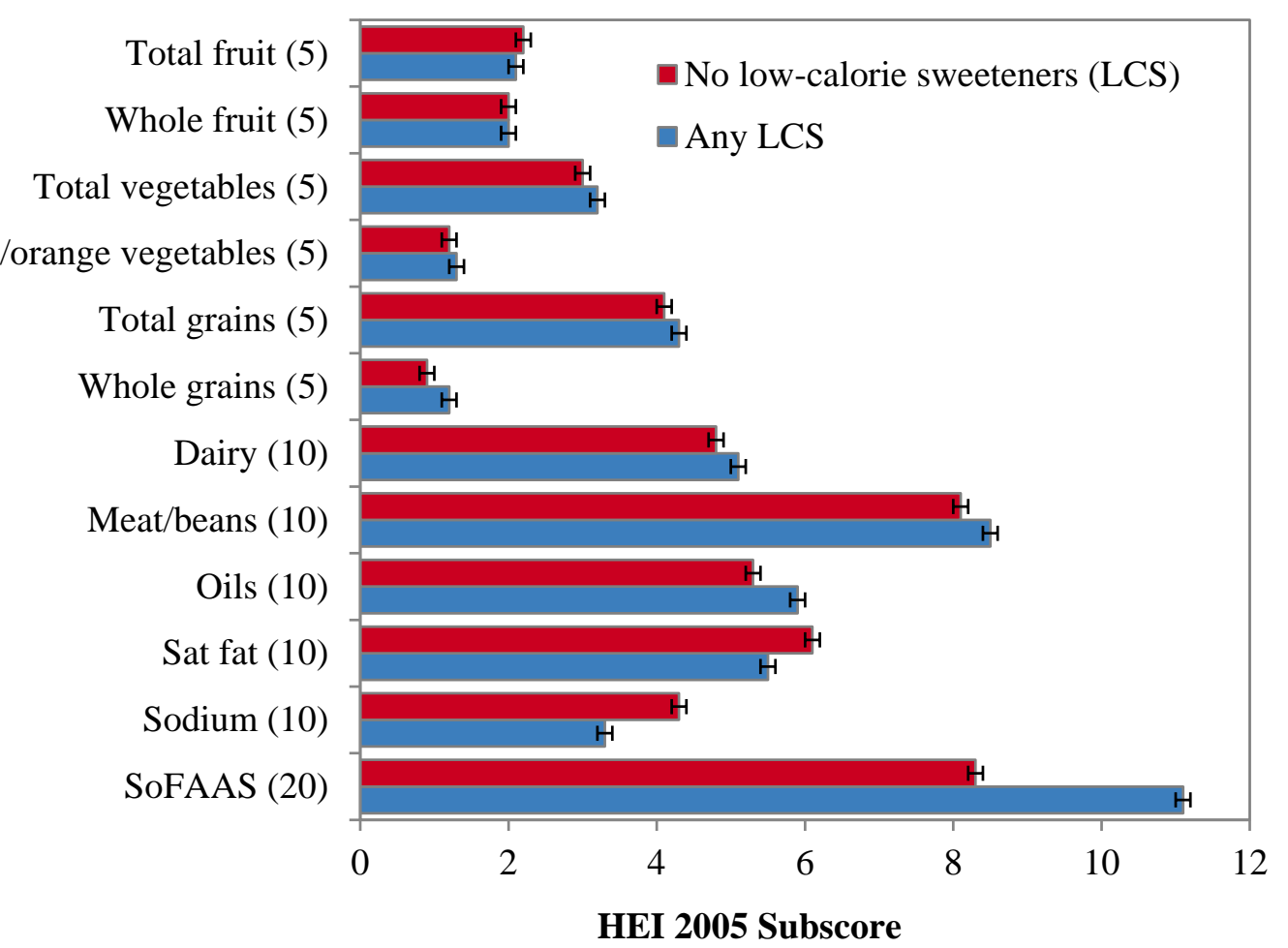


Figure 2. Adjusted mean Healthy Eating Index 2005 (HEI) subscores among non low-calorie sweetener (LCS) users, and LCS use category. Analysis adjusted for age group, race/ethnicity and gender.

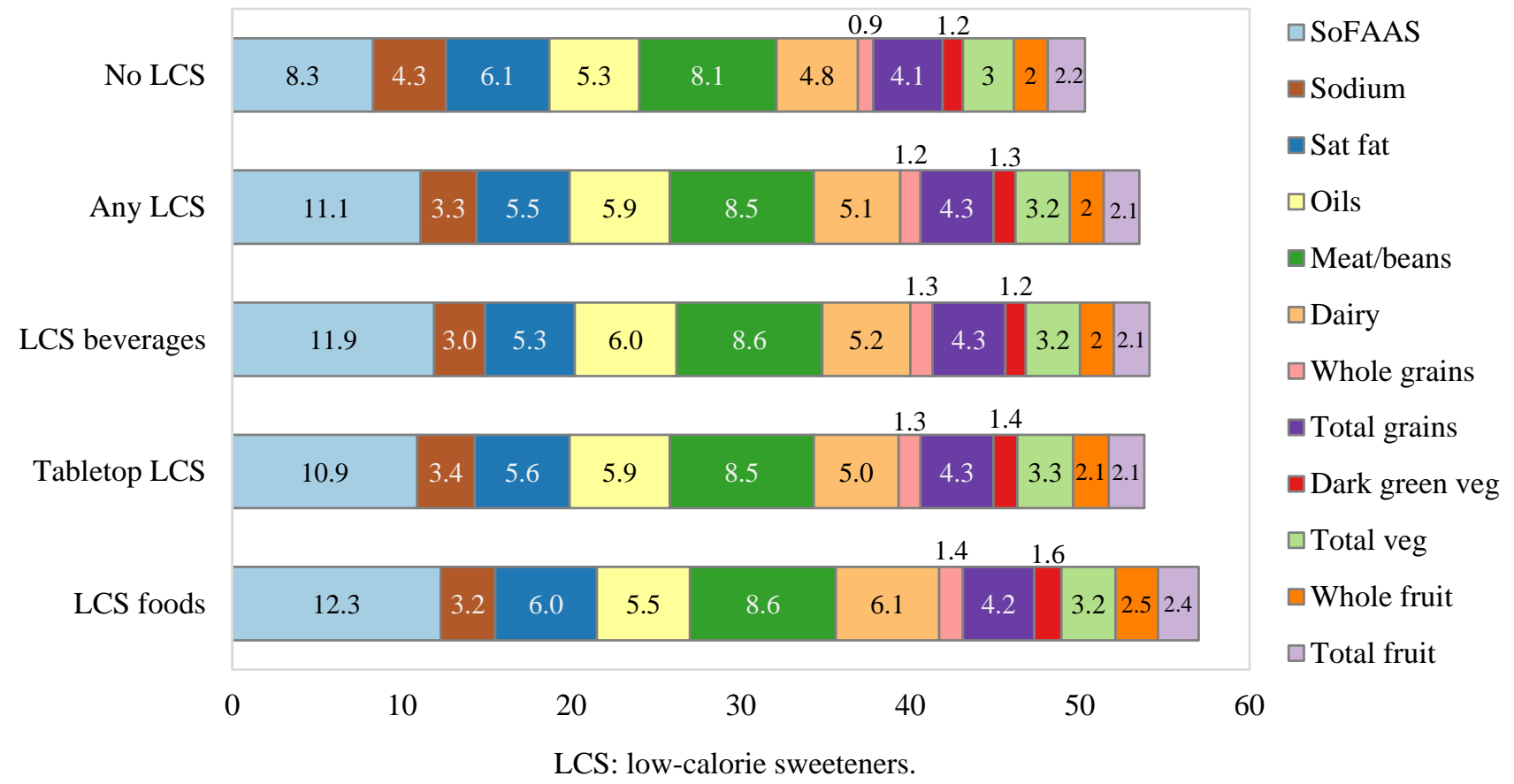

On the other hand, LCS consumers in every product category had lower HEI subscores for sodium, indicating higher consumption $(p<0.001)$. With the exception of LCS foods, LCS consumers also had lower saturated fat subscores, indicating higher consumption $(p<0.001)$.

\subsection{LCS Use and Health Behaviors}

Figure 3 shows that LCS consumers were more physically active than were non-consumers. The association held after adjusting for age group, gender, family income and race/ethnicity (results not shown). LCS consumers were $15 \%$ more likely to be very active compared to non-consumers after adjusting for age only. After adjusting for gender, income and race/ethnicity, LCS consumers were still $10 \%$ more likely to be physically active. Those results suggest that the observed link between LCS consumption and higher physical activity cannot be explained simply by differences in SES.

Approximately $20.7 \%$ of LCS consumers were current smokers as compared to $26.2 \%$ of non-LCS consumers, after adjusting for age group, gender, family income and race/ethnicity. More LCS consumers were former smokers. LCS consumers consumed alcohol less often, though the difference was not profound.

Table 3 shows the health behaviors by LCS product category. LCS beverage consumers were least likely to be sedentary and were more likely to be highly active than were non consumers (in the third or fourth quartiles of Metabolic Equivalent of Task (MET) values for physical activity). LCS beverage consumers were less likely to be current smokers than were non consumers; however, they were more likely to be former smokers. LCS beverage consumers tended to consume less alcohol than did non consumers. Generally, the relation between LCS beverage consumption and health behaviors was similar for both men and women (data not shown). 
Figure 3. Age-adjusted prevalence of health behaviors among consumers of any low-calorie sweetener (LCS) compared to non-consumers, NHANES 1999-2008. Data are for physical activity (left) and for smoking status (right).
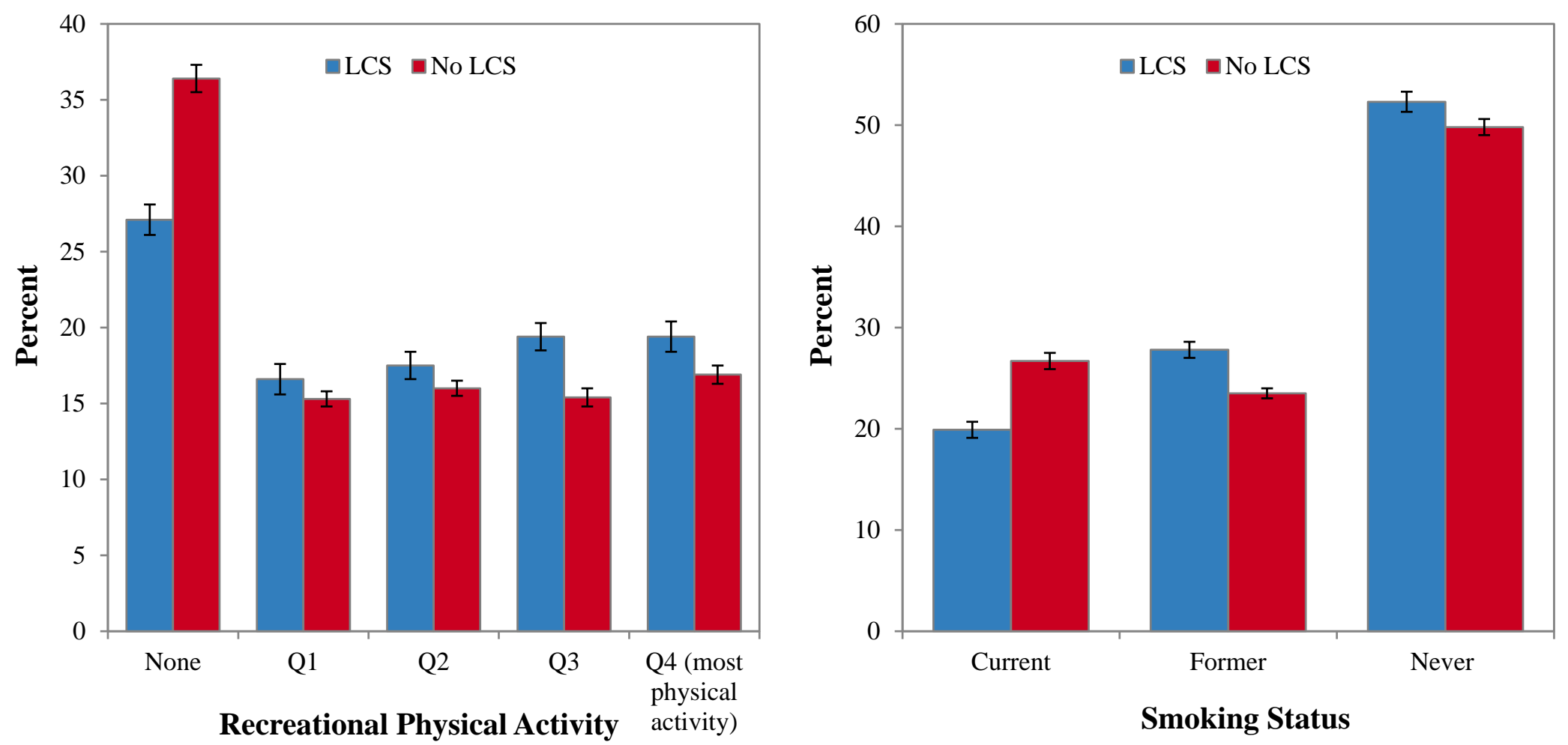

LCS: low-calorie sweeteners. 
Table 3. Age-adjusted prevalence (or means) of health behaviors among consumers of low-calorie sweetener (LCS) beverages, tabletop LCS and LCS foods, NHANES 1999-2008.

\begin{tabular}{|c|c|c|c|c|c|c|c|c|c|c|}
\hline & $n$ & $\begin{array}{c}\text { LCS } \\
\text { beverages }\end{array}$ & $\begin{array}{c}\text { No LCS } \\
\text { beverages }\end{array}$ & $p$-value & $\begin{array}{c}\text { Tabletop } \\
\text { LCS }\end{array}$ & $\begin{array}{c}\text { No tabletop } \\
\text { LCS }\end{array}$ & $p$-value & LCS food & $\begin{array}{c}\text { No } \\
\text { LCS } \\
\text { food }\end{array}$ & $p$-value \\
\hline \multicolumn{11}{|l|}{$\begin{array}{l}\text { Recreational moderate/vigorous } \\
\text { physical activity }^{1}\end{array}$} \\
\hline None & 6630 & $26.2(1.2)$ & $35.5(0.8)$ & & $26.6(1.5)$ & $34.4(0.8)$ & & $23.2(2.1)$ & $34.3(0.8)$ & \\
\hline Q1 & 2359 & $17.3(1.5)$ & $15.4(0.4)$ & & $15.6(1.2)$ & $15.7(0.5)$ & & $15.0(2.1)$ & $15.7(0.5)$ & \\
\hline Q2 & 2377 & $18.2(1.2)$ & $16.1(0.5)$ & $<0.001$ & $17.8(1.5)$ & $16.3(0.4)$ & $<0.001$ & $17.9(2.2)$ & $16.3(0.4)$ & $<0.001$ \\
\hline Q3 & 2370 & $19.3(1.1)$ & $16.0(0.6)$ & & $18.2(1.4)$ & $16.5(0.5)$ & & $22.7(2.5)$ & $16.5(0.5)$ & \\
\hline Q4 (most physical activity) & 2385 & $19.1(1.2)$ & $17.0(0.6)$ & & $21.8(1.9)$ & $17.2(0.6)$ & & $21.3(2.8)$ & $17.2(0.6)$ & \\
\hline \multicolumn{11}{|l|}{ Smoking status } \\
\hline Current smoker & 4997 & $18.7(1.0)$ & $26.0(0.7)$ & & $20.7(1.6)$ & $25.2(0.6)$ & & $15.1(1.8)$ & $25.1(0.6)$ & \\
\hline Former smoker & 5861 & $28.4(1.2)$ & $24.0(0.5)$ & $<0.001$ & $27.7(1.2)$ & $24.3(0.4)$ & $<0.001$ & $25.8(2.1)$ & $24.8(0.4)$ & $<0.001$ \\
\hline Never smoker & 11,349 & $52.9(1.3)$ & $50.0(0.7)$ & & $51.6(1.6)$ & $50.5(0.7)$ & & $59.1(2.6)$ & $50.2(0.7)$ & \\
\hline \multicolumn{11}{|l|}{ Frequency of alcohol consumption } \\
\hline None or $<12$ drinks in past year & 7477 & $28.1(1.4)$ & $29.9(1.1)$ & & $26.8(1.4)$ & $29.7(1.2)$ & & $29.2(2.6)$ & $29.6(1.1)$ & \\
\hline$<0.2$ drinks/wk & 3504 & $16.6(0.8)$ & $16.3(0.5)$ & & $18.1(1.5)$ & $16.2(0.4)$ & & $18.8(1.8)$ & $16.3(0.4)$ & \\
\hline $0.2-0.99$ drinks/wk & 3226 & $18.8(1.0)$ & $16.3(0.4)$ & 0.045 & $17.6(1.6)$ & $16.6(0.4)$ & $<0.001$ & $17.7(2.0)$ & $16.6(0.4)$ & $<0.001$ \\
\hline 1-5.99 drinks/wk & 3391 & $19.8(1.3)$ & $18.3(0.6)$ & & 19.5 (1.6) & $18.5(0.6)$ & & $20.1(2.6)$ & $18.6(0.6)$ & \\
\hline$\geq 6$ drinks/wk & 3440 & $16.7(1.1)$ & $19.2(0.6)$ & & $18.1(1.8)$ & $19.0(0.6)$ & & $14.2(1.7)$ & $18.9(0.8)$ & \\
\hline Grams of alcohol (mean) 2,3 & - & $11.2(0.6)$ & $10.7(0.4)$ & 0.64 & $10.2(0.6)$ & $10.8(0.4)$ & 0.29 & $9.4(0.8)$ & $10.8(0.4)$ & 0.08 \\
\hline
\end{tabular}

${ }^{1}$ Data from 1999-2006 NHANES, since Metabolic Equivalent of Task (MET) values were not collected in the 2007-2008 cycle of NHANES. ${ }^{2}$ Value represents the mean. ${ }^{3}$ From $24-$ hour recall; adjusted for energy, gender and age group; LCS: low-calorie sweeteners. 
Tabletop LCS consumers were more physically active and were less likely to be current smokers than were non-consumers. Tabletop LCS consumers did not differ in alcohol intakes from non-consumers, though they were slightly less likely to be never or infrequent alcohol drinkers. Similar trends were obtained for consumers of LCS foods. LCS food consumers were much less likely to be current smokers than were non-consumers.

\section{Discussion}

The present study extends prior research by providing the first analyses of the association of LCS use with diet quality measures based on the Healthy Eating Index 2005. Past authors [5] have called for further research on the impact of diet beverages on diet quality, with particular attention to sugar consumption $[4,5]$. One concern has been that the use of LCS beverages was likely to be associated with higher, as opposed to lower, sugar consumption and with lower-quality diets [4].

The present findings were that LCS users had significantly higher quality diets than did non-users. That observation held for every LCS product category. The improvement in the total HEI 2005 scores was largely driven by more favorable SoFAAS subscores. The SoFAAS component tracks the consumption of solid fats, added sugars and alcohol, the principal sources of "empty calories" [21] in the American diet. For every product category, LCS use was associated with higher SoFAAS subscores, indicating lower consumption of solid fats, alcohol and added sugars.

Additional analyses (Table 3) showed that LCS use was not systematically associated with amount of alcohol consumed from a 24-h recall. Amounts of alcohol consumed were comparable across LCS users and non-users for every LCS product category. The consumption of saturated fat was moderately elevated among LCS consumers. The conclusion is that improved SoFAAS subscores were largely driven by a drop in added sugars consumption among LCS users as opposed to non-users.

The present study used Federal measures of diet quality to counter some interpretations of past research [5]. In one study [5], researchers compared the consumption of selected sweet snacks, such as ice cream, dairy desserts, sweet rolls, cakes, pastries, cookies, pies and candy (696 items) by users of sugar sweetened (SSB) and diet beverages. The observation that both groups derived approximately $11 \%-12 \%$ of their energy intake (238 kcal/day) from sweet snacks was interpreted to mean that the sweet taste of LCS enhanced appetite and encouraged sugar craving and dependence [5,22]. The present analyses do not support that interpretation.

In this study, diet quality was measured using HEI-2005, a 12-component tool designed to measure compliance with dietary recommendations and guidelines. As expected, LCS consumers had more favorable SoFAAS subscores, a measure specifically designed to track the consumption of empty calories. The present data clearly showed that SoFAAS subscores applied to the total diet were significantly higher for LCS users than for non-users, across all product categories, and the association held upon adjustment for covariates. The present conclusion is that the amount of added sugar in the diets of LCS users was, in fact, lower. In general, the consumption of added sugars in U.S. diets has decreased [23,24], whereas the consumption of LCS has increased [14].

Consumers of LCS foods showed the greatest differences in HEI scores. Though fewer adults consumed LCS foods, the difference in HEI scores between consumers and non-consumers was greater than for LCS beverages or for tabletop LCS. Consumers of LCS foods had HEI scores that were about 
$12 \%$ higher than non-consumers. Consumers of LCS foods also tended to consume more fruits, vegetables, whole grains, meat/beans and fewer calories from SoFAAS. These data suggest that consumers of LCS foods may be more likely to adopt a prudent diet when compared to consumers of other LCS products.

LCS consumers were less sedentary, exercised more and smoked less than did non-consumers. These positive health behaviors were observed for all product categories: tabletop sweeteners, diet beverages and low calorie foods. One possible explanation is that LCS use may be a proxy marker for higher quality diets and for better health behaviors.

This study had several strengths. First, analyses were based on a large, nationally-representative sample of United States adults. Results are thus generalizable on a population level and can be compared to other recent studies. Second, this was one of the first studies to assign LCS consumers to different product categories. With few exceptions [14], most of the literature has dealt exclusively with diet beverages. Given differences in LCS use by age group and diminishing consumption of carbonated beverages later in life, this classification scheme may improve our understanding of the contextual, behavioral and environmental influences on LCS consumption by SES and demographic variables.

The use of HEI 2005 and its subscores was another strength. Previous studies have called for more research on the overall diet quality of LCS consumers. The HEI 2005 is an accepted metric of diet quality, and has been used in numerous studies evaluating the diet quality of the population. Evaluating diet quality by LCS using alternative metrics is one potential area of future research.

Several limitations should be noted. Owing to the relatively low use of tabletop LCS and LCS foods, the accuracy of consumption data for these products is not well established. Different types of LCS (i.e., sucralose or saccharin) could not be identified in the nutrient composition database. As new LCS foods enter the marketplace, the developers of food composition databases ought to consider the feasibility of explicitly identifying beverages and foods containing LCS.

\section{Conclusions}

The use of diet products, reduced in added sugars, remains a popular strategy for weight control [25], one that has been tested in numerous studies [26,27]. The present analyses suggest that LCS consumers may differ in several, previously unobserved, ways from non-consumers in terms of their health behaviors. In particular, LCS consumers were more physically active and had higher HEI scores. In addition to the benefits conferred by the reduction of energy from added sugars, LCS consumption may be a marker for other positive health behaviors and lifestyles

\section{Acknowledgments}

The authors would like to thank Wesley Tang for his assistance preparing and formatting this manuscript.

\section{Author Contributions}

Adam Drewnowski and Colin D. Rehm designed the analyses. Colin D. Rehm conducted the analyses. Both Adam Drewnowski and Colin D. Rehm wrote the manuscript. 


\section{Appendix}

Table A1. Adjusted association between any low-calorie sweetener (LCS) consumption and the Healthy Eating Index 2005 (HEI) score and the HEI components. Analysis adjusted for age group, race/ethnicity and gender.

\begin{tabular}{lcccc}
\hline & $\begin{array}{c}\text { Maximum } \\
\text { Score }\end{array}$ & Any LCS & No LCS & $\begin{array}{c}\boldsymbol{p} \text {-value of } \\
\text { Difference }\end{array}$ \\
\hline Healthy Eating Index (2005) & 100 & $53.6(0.3)$ & $50.4(0.3)$ & $<0.001$ \\
HEI 1: Total fruit & 5 & $2.1(0.1)$ & $2.2(0.1)$ & 0.08 \\
HEI 2: Whole fruit & 5 & $2.0(0.1)$ & $2.0(0.1)$ & 0.38 \\
HEI 3: Total vegetables & 5 & $3.2(0.1)$ & $3.0(0.1)$ & $<0.001$ \\
HEI 4: Dark green/orange veg and legumes ${ }^{2}$ & 5 & $1.3(0.1)$ & $1.2(0.1)$ & 0.15 \\
HEI 5: Total grains & 5 & $4.3(0.1)$ & $4.1(0.1)$ & $<0.001$ \\
HEI 6: Whole grains & 5 & $1.2(0.1)$ & $0.9(0.1)$ & $<0.001$ \\
HEI 7: Dairy/milk & 10 & $5.1(0.1)$ & $4.8(0.1)$ & $<0.001$ \\
HEI 8: Meat and beans & 10 & $8.5(0.1)$ & $8.1(0.1)$ & $<0.001$ \\
HEI 9: Oils & 10 & $5.9(0.1)$ & $5.3(0.1)$ & $<0.001$ \\
HEI 10: Saturated fat & 10 & $5.5(0.1)$ & $6.1(0.1)$ & $<0.001$ \\
HEI 11: Sodium & 10 & $3.3(0.1)$ & $4.3(0.1)$ & $<0.001$ \\
HEI 12: SoFAAS * & 20 & $11.1(0.1)$ & $8.3(0.1)$ & $<0.001$ \\
HEI-2005 (no SoFAAS) & 80 & $42.5(0.2)$ & $42.1(0.2)$ & 0.10 \\
\hline
\end{tabular}

${ }^{1}$ Legumes are included in this category if they were not needed to meet the meat and bean component score; * Calories from solid fat, alcoholic beverages and added sugars (SoFAAS); LCS: low-calorie sweetener. 
Table A2. Adjusted association between any low-calorie sweetener (LCS), tabletop LCS and LCS foods consumption and the Healthy Eating Index 2005 (HEI) score and the HEI components. Analysis adjusted for age group, race/ethnicity and gender.

\begin{tabular}{|c|c|c|c|c|c|c|c|c|c|c|}
\hline & $\begin{array}{l}\text { Max } \\
\text { Score }\end{array}$ & $\begin{array}{c}\text { LCS } \\
\text { Beverages }\end{array}$ & $\begin{array}{c}\text { No LCS } \\
\text { Beverages }\end{array}$ & $p$-value & $\begin{array}{c}\text { Tabletop } \\
\text { LCS }\end{array}$ & $\begin{array}{c}\text { No Tabletop } \\
\text { LCS }\end{array}$ & $p$-value & $\begin{array}{c}\text { LCS } \\
\text { Foods }\end{array}$ & $\begin{array}{l}\text { No LCS } \\
\text { Foods } \\
\end{array}$ & $p$-value \\
\hline Healthy Eating Index (2005) & 100 & $54.3(0.40)$ & $50.7(0.27)$ & $<0.001$ & $53.8(0.45)$ & $51.1(0.26)$ & $<0.001$ & $57.1(0.7)$ & $51.1(0.2)$ & $<0.001$ \\
\hline HEI 1: Total fruit & 5 & $2.1(0.06)$ & $2.2(0.04)$ & 0.20 & $2.1(0.06)$ & $2.2(0.04)$ & 0.61 & $2.4(0.1)$ & $2.2(0.04)$ & 0.004 \\
\hline HEI 2: Whole fruit & 5 & $2.0(0.06)$ & $2.0(0.04)$ & 0.31 & $2.1(0.06)$ & $2.0(0.03)$ & 0.19 & $2.5(0.1)$ & $2.0(0.04)$ & $<0.001$ \\
\hline HEI 3: Total vegetables & 5 & $3.2(0.04)$ & $3.1(0.02)$ & $<0.001$ & $3.3(0.04)$ & $3.1(0.02)$ & $<0.001$ & $3.2(0.1)$ & $3.1(0.02)$ & 0.10 \\
\hline $\begin{array}{l}\text { HEI4: Dark green/orange } \\
\text { vegetables and legumes }{ }^{1}\end{array}$ & 5 & $1.2(0.04)$ & $1.3(0.03)$ & 0.55 & $1.4(0.06)$ & $1.2(0.03)$ & $<0.001$ & $1.6(0.1)$ & $1.2(0.03)$ & $<0.001$ \\
\hline HEI 5: Total grains & 5 & $4.3(0.03)$ & $4.1(0.02)$ & $<0.001$ & $4.3(0.04)$ & $4.2(0.01)$ & 0.01 & $4.2(0.16)$ & $4.2(0.02)$ & 0.80 \\
\hline HEI 6: Whole grains & 5 & $1.3(0.04)$ & $1.0(0.02)$ & $<0.001$ & $1.3(0.05)$ & $1.0(0.02)$ & $<0.001$ & $1.4(0.1)$ & $1.0(0.02)$ & 0.01 \\
\hline HEI 7: Dairy/milk & 10 & $5.2(0.08)$ & $4.8(0.05)$ & $<0.001$ & $5.0(0.10)$ & $4.9(0.05)$ & 0.13 & $6.1(0.2)$ & $4.8(0.05)$ & $<0.001$ \\
\hline HEI 8: Meat and beans & 10 & $8.6(0.05)$ & $8.1(0.03)$ & $<0.001$ & $8.5(0.07)$ & $8.2(0.03)$ & $<0.001$ & $8.6(0.1)$ & $8.2(0.03)$ & 0.005 \\
\hline HEI 9: Oils & 10 & $6.0(0.08)$ & $5.3(0.05)$ & $<0.001$ & $5.9(0.11)$ & $5.4(0.04)$ & $<0.001$ & $5.5(0.2)$ & $5.4(0.04)$ & 0.61 \\
\hline HEI 10: Saturated fat & 10 & $5.3(0.11)$ & $6.1(0.05)$ & $<0.001$ & $5.6(0.11)$ & $6.0(0.05)$ & $<0.001$ & $6.0(0.2)$ & $5.9(0.05)$ & 0.59 \\
\hline HEI 11: Sodium & 10 & $3.0(0.06)$ & $4.3(0.04)$ & $<0.001$ & $3.4(0.09)$ & $4.1(0.04)$ & $<0.001$ & $3.2(0.1)$ & $4.1(0.04)$ & $<0.001$ \\
\hline HEI 12: SoFAAS * & 20 & $11.9(0.18)$ & $8.5(0.12)$ & $<0.001$ & $10.9(0.2)$ & $8.9(0.1)$ & $<0.001$ & $12.3(0.3)$ & $9.0(0.10)$ & $<0.001$ \\
\hline
\end{tabular}

${ }^{1}$ Legumes are includes in this category if they were not needed to meet the meat and bean component score; * Calories from solid fats, alcohol and added sugars (SoFAAS); LCS: low-calorie sweetener. 


\section{Conflicts of Interest}

Adam Drewnowski has received grants, contracts, honoraria and consulting fees from numerous food and beverage companies and other commercial and nonprofit entities with interests in diet quality and health. The University of Washington has received grants, donations and contracts from both the public and the private sector. Colin D. Rehm declares no conflict of interest.

\section{References and Notes}

1. Anderson, G.; Foreyt, J.; Sigman-Grant, M.; Allison, D.B. The use of low-calorie sweeteners by adults: Impact on weight management. J. Nutr. 2012, 142, 1163-1169.

2. Bellisle, F.; Drewnowski, A. Intense sweeteners, energy intake and the control of body weight. Eur. J. Clin. Nutr. 2007, 61, 691-700.

3. Gardner, C.; Wylie-Rosett, J.; Gidding, S.S.; Steffen, L.M.; Johnson, R.K.; Reader, D.; Lichtenstein, A.H. Nonnutritive sweeteners: Current use and health perspectives: A scientific statement from the American Heart Association and the American Diabetes Association. Diabetes Care 2012, 35, 1798-1808.

4. Ludwig, D.S. Artificially sweetened beverages: Cause for concern. JAMA 2009, 302, 2477-2478.

5. Bleich, S.N.; Wolfson, J.A.; Vine, S.; Wang, Y.C. Diet-beverage consumption and caloric intake among US adults, overall and by body weight. Am. J. Public Health 2014, 104, 72-78.

6. Bellisle, F.; Altenburg de Assis, M.A.; Fieux, B.; Preziosi, P.; Galan, P.; Guy-Grand, B.; Hercberg, S. Use of 'light' foods and drinks in French adults: Biological, anthropometric and nutritional correlates. J. Hum. Nutr. Diet. 2001, 14, 191-206.

7. Blackburn, G.L.; Kanders, B.S.; Lavin, P.T.; Keller, S.D.; Whatley, J. The effect of aspartame as part of a multidisciplinary weight-control program on short- and long-term control of body weight. Am. J. Clin. Nutr. 1997, 65, 409-418.

8. Fakhouri, T.H.; Kit, B.K.; Ogden, C.L. Consumption of diet drinks in the United States, 2009-2010. NCHS Data Brief. 2012, 109, 1-8.

9. Drewnowski, A.; Rehm, C.D.; Constant, F. Water and beverage consumption among adults in the United States: Cross-sectional study using data from NHANES 2005-2010. BMC Public Health 2013, 13, 1068.

10. Rehm, C.D.; Monsivais, P.; Drewnowski, A. The quality and monetary value of diets consumed by adults in the United States. Am. J. Clin. Nutr. 2011, 94, 1333-1339.

11. Ford, E.S.; Merritt, R.K.; Heath, G.W.; Powell, K.E.; Washburn, R.A.; Kriska, A.; Haile, G. Physical activity behaviors in lower and higher socioeconomic status populations. Am. J. Epidemiol. 1991, 133, 1246-1256.

12. Giles-Corti, B.; Donovan, R.J. Socioeconomic status differences in recreational physical activity levels and real and perceived access to a supportive physical environment. Prev. Med. 2002, 35, 601-611.

13. Sigman-Grant, M.; Hsieh, G. Reported use of reduced-sugar foods and beverages reflect high-quality diets. J. Food Sci. 2005, 70, 43-46. 
14. Sylvetsky, A.C.; Welsh, J.A.; Brown, R.J.; Vos, M.B. Low-calorie sweetener consumption is increasing in the United States. Am. J. Clin. Nutr. 2012, 96, 640-646.

15. Ng, S.; Slining, M.; Popkin, B. Use of caloric and noncaloric sweeteners in US consumer packaged foods, 2005-2009. J. Acad. Nutr. Diet. 2012, 112, 1828-1834,

16. Guenther, P.M.; Reedy, J.; Krebs-Smith, S.M. Development of the Healthy Eating Index-2005. J. Am. Diet. Assoc. 2008, 108, 1896-1901.

17. Centers for Disease Control and Prevention (CDC); National Center for Health Statistics. About the National Health and Nutrition Exmaination Survey (NHANES). Available online: Http://www.cdc.gov/nchs/nhanes/about_nhanes.htm (accessed on 22 April 2014).

18. Centers for Disease Control and Prevention (CDC); National Center for Health Statistics. NCHS Research Ethics Review Board (ERB) Approval. Available online: Http://www.cdc.gov/nchs/ nhanes/irba98.htm (accessed on 15 January 2013).

19. Centers for Disease Control and Prevention; National Center for Health Statistics. National Health and Nutrition Examination Survey. Available online: Http://www.cdc.gov/nchs/tutorials/dietary/ SurveyOrientation/DietaryDataOverview/Info2.htm (accessed on 30 March 2014).

20. Ahuja, J.K.A.; Montville, J.B.; Omolewa-Tomobi, G.; Heendeniya, K.Y.; Martin, C.L.; Steinfeldt, L.C.; Anand, J.; Adler, M.E.; LaComb, R.P.; Moshfegh, A.J. USDA Food and Nutrient Database for Dietary Studies, 5.0; U.S. Department of Agriculture, Agricultural Research Service, Food Surveys Research Group: Beltsville, MD, USA, 2012.

21. Guenther, P.M.; Casavale, K.O.; Reedy, J.; Kirkpatrick, S.I.; Hiza, H.A.; Kuczynski, K.J.; Kahle, L.L.; Krebs-Smith, S.M. Update of the Healthy Eating Index: HEI-2010. J. Acad. Nutr. Diet. 2013, 113, 569-580.

22. Fowler, S.P.; Williams, K.; Resendez, R.G.; Hunt, K.J.; Hazuda, H.P.; Stern, M.P. Fueling the obesity epidemic? Artificially sweetened beverage use and long-term weight gain. Obesity (Silver Spring) 2008, 16, 1894-1900.

23. Ervin, R.B.; Ogden, C.L. Consumption of added sugars among U.S. adults, 2005-2010. NCHS Data Brief. 2013, 122, 1-8.

24. Welsh, J.A.; Sharma, A.J.; Grellinger, L.; Vos, M.B. Consumption of added sugars is decreasing in the United States. Am. J. Clin. Nutr. 2011, 94, 726-734.

25. Fitch, C.; Keim, K.S. Position of the Academy of Nutrition and Dietetics: Use of nutritive and nonnutritive sweeteners. J. Acad. Nutr. Diet. 2012, 112, 739-758.

26. Ebbeling, C.B.; Feldman, H.A.; Osganian, S.K.; Chomitz, V.R.; Ellenbogen, S.J.; Ludwig, D.S. Effects of decreasing sugar-sweetened beverage consumption on body weight in adolescents: A randomized, controlled pilot study. Pediatrics 2006, 117, 673-680.

27. Stookey, J.D.; Constant, F.; Gardner, C.D.; Popkin, B.M. Replacing sweetened caloric beverages with drinking water is associated with lower energy intake. Obesity (Silver Spring) 2007, 15, 3013-3022.

(C) 2014 by the authors; licensee MDPI, Basel, Switzerland. This article is an open access article distributed under the terms and conditions of the Creative Commons Attribution license (http://creativecommons.org/licenses/by/4.0/). 